\title{
Iris Recognition from an Image at Lengthy Distance by using Deep Belief Neural Network (DBN)
}

\author{
Swati D. Shirke, C.Rajabhushanam
}

\begin{abstract}
Now a days, biometric is a one of the best method which is used for the detection of person is iris recognition. A large portion of different frameworks are equally introduces for individual ID like as distinguishing proof cards or tokens, mystery codes, passwords, and so on. Yet, the issues of these sort of frameworks are, the mystery codes and passwords can be split, the recognizable proof cards can be harmed. Subsequently the successful strategy for the individual recognizable proof is vital. Iris gives the unmistakable data about an individual. Iris recognition is the process of identifying persons automatically using their iris. Iris provides the distinctive information about a person. This paper exhibits the deep learning-based methodology for the iris acknowledgment. Firstly, the picture is pre-handled to get the precise area of the iris. From that point onward, iris locale is extricated utilizing Hough Transform, which is pursued with the division and standardization of the iris area utilizing the Daugman's Rubber sheet model. When the division is played out, the features are separated by utilizing the Local Gradient Pattern (LGP) and ScaT-LOOP that is the mixture of Scattering transforms (ST), Tetrolet transforms (TT), and Local Optimal Oriented Pattern (LOOP) descriptors. At last, steepest slope based Deep Belief Neural Network (DBN) is used for the iris acknowledgment. The exhibition of iris acknowledgment utilizing the DBN classifier is assessed regarding precision, False Rejection Rate (FRR) and False Acceptance Rate (FAR). The proposed iris acknowledgment strategy accomplishes the most extreme precision of $97.96 \%$, negligible FAR of 0.493\%, and insignificant FRR of 0.48\% that shows its predominance.
\end{abstract}

Keywords-Iris recognition, Deep Belief Network(DBN), Daugman's rubber sheet model, Local Gradient pattern, biometrics. Back Propagation Neural Network, Cronologival Neural Network.

\section{INTRODUCTION}

In the ongoing years, biometrics assumes a significant job in security frameworks. Biometrics is classified into picture based frameworks and sign based frameworks. Biometrics is an instrument for separating subjects in a dependable way utilizing conduct or physical attributes [9]. Presently a days, in world a large portion of the nations faces the issue of fake character. This is an intense issue and it is very hard to distinguish the false individual. Be that as it may, the biometric framework can tackle the above issues.

Revised Manuscript Received on July 10, 2019.

Ms. Swati D. Shirke, Ph.D.Scholor, Bharath Institute of Higher Education and Research Chennai, Tamil Nadu, India .Email:shirke.swati14@gmail.com

Dr. C.Rajabhushanam, Professor, Computer Science \& Engineering, Bharath Institute of Higher Education and Research, Chennai, Tamil Nadu, India. rajabhushanamc.cse@bharathuniv.ac.in
Along these lines the greater part of the nations utilizes biometric framework for security reason with the end goal that in custom scope, air terminal boarding, gathering passageway, etc. The Indian government additionally utilizes biometric framework for distinguishing proof of resident in various applications like as in rashan shop, Aadhar venture, in various government test structures and enrollment dept. and so forth. There are various sorts of biometrics are available, for example, voice, palm, finger, face, DNA, and so on. Be that as it may, the iris acknowledgment is the most precise and stable biometric framework for individual distinguishing proof. Since the Iris is a special thing of an individual, it doesn't change with time and condition. It stays fixed and consistent for the duration of the life of individual. Likewise the blunder rate, materialness and exactness of iris acknowledgment framework superior to the next biometric frameworks. Along these lines for accomplishing and looking after security, the iris acknowledgment framework assumes a significant job.

Different methodologies are utilized for the limitation of iris, similar to Distance Regularized Level Set Evolution (DRLSE), integral differential administrator, Circular Hough Transform (CHT), and Active Contour (AC). Tsai [17] utilizes fluffy coordinating system to distinguish the element of iris focuses. Here, the closeness score table is used to look at the component focuses in coordinating calculations. Counterfeit neural systems (ANNs) are planned by natural neural systems for assessing or approximating capacities that depends on a few sources of info, which is obscure. ANNs accomplished better outcomes in forecast, arrangement, design acknowledgment and control, and order. ANN contains a gathering of fake neurons, which registers and controls the information dependent on the connectionist strategy. A few looks into presented neural systems for iris acknowledgment [18]. Signal-based frameworks contain distinguishing proof of ECG just as the identification of speaker. Picture based frameworks comprise of motion, manually written mark, voice, hand geometry, step, iris acknowledgment, and face [1]. These days, high goals iris pictures are caught utilizing the cell phones [3]. Over the most recent couple of decades, the iris should guarantee greatest precision, and there has been a blast of enthusiasm for iris acknowledgment [5]. At the point when contrasted with old style security techniques (counting secret word, card, PIN, and key)

Putigence Engineering \& Sciences Publication 
this innovation makes different advantages, for example, extraordinary recognizable proof of people, hard to fashion, easy to use, consistently with the client (no outer conveying), secure, and versatile.

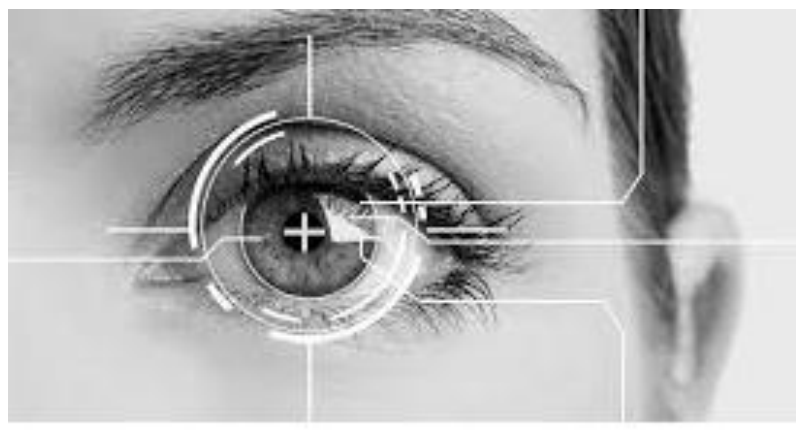

Fig. 1.Sample of iris recognisation.

The way toward perceiving the people or articles naturally dependent on biometric information is named as biometric acknowledgment framework (BRS) [9]. This paper proposes the acknowledgment of iris utilizing profound learning-based methodology. From the start, the information picture is sustained to the pre-preparing to get the careful locale of the iris. After pre-preparing, the district extraction is performed by applying Hough Transform (HT). In the wake of separating the area, the division of iris is finished utilizing the Daugman's rubber sheet model, which is pursued with the component extraction by utilizing the Local Gradient Pattern (LGP) and the ScatT-LOOP descriptors. After the extraction of iris includes, the DBN is utilized for the acknowledgment of iris.

The association of the paper is: Section 2 gives the Writing review of different existing techniques for iris acknowledgment. Area 3 talks about the methods used in the proposed system. Segment 4 is arrangements of building block diagram of projected structure by utilizing DBN. The outcomes and talks are clarified in area 5, lastly, segment 6 represents the conclusion of paper.

\section{Writing REVIEW}

This segment manages current techniques for iris acknowledgment clarified as pursues:

Abhishek Gangwar, Akanksha Joshi [25] created Deep Iris Net for iris portrayal. The methodology was intended for ideal iris portrayal, and better usage of figuring assets. The strategy did not think about different highlights for better framework execution. Ashok K Bhateja et al. Ahmadi and Gholamreza Akbarizadeh [22] built up a methodology by coordinating the Particle Swarm Optimization (PSO) and multi-layer perceptron Neural Network $(\mathrm{NN})$ to improve the speculation execution. Gabor include extraction was acquainted with concentrate the highlights on the iris pictures. Soubhagya Sankar Barpanda et al.[24] introduced a strategy for separating the component of iris-dependent on tunable channel bank. At that point, the districts were expelled dependent on integrodifferential administrator. When the territory of extraction was played out, the channel bank was presented for expelling highlights from the standardized pictures. Bi-symmetrical channels were not considered for high-recurrence selectivity. N. Pattabhi Ramaiah and Ajay Kumar [19] presented Naive Bayes Nearest Neighbor (NBNN) classifier to figure the iris designs from the iris pictures. This structure utilized bi-phantom acknowledgment framework to get the week time window for it.

Obvious and infra-red pictures, which perform iris coordinating from different areas. The strategy neglected to improve coordinating precision for iris acknowledgment. Chun-Wei Tan, and Ajay Kumar [20] built up an iris encoding plan for giving individual distinguishing proof ability to the iris pictures. The encoded iris highlight permitted the iris layout coordinating dependent on the Hamming separation, which was spoken to in parallel structure. Confined iris highlights were not considered for better execution. Kien Nguyen et al. [21] created Convolutional Neural Networks (CNNs) for speaking to the attributes of the picture. The textural subtleties were removed and encoded utilizing gabor wavelets, and change the phasor reaction dependent on twofold code. The technique does not boost the limit of the iris formats. Neda The structure neglected to consolidate PSO with a fluffy framework. Kiran B. Raja et al.[23] created multi-fix profound highlights dependent on profound scanty channels for solid iris acknowledgment. This system was used for characterization through amplified probability, even under single example enrolment. [26] planned an effective iris acknowledgment model dependent on k-closest subspace (portions), and compressive detecting. K-closest subspace technique was utilized for short listing the classes to moderate the time. At that point, the shortlisted up-and-comers were parceled into areas, after that the scanty acknowledgment was connected to each part. Here, Cumulative Sparse Concentration Index (CSCI)- based classifiers, Sector-based classifier, and k-closest separation classifier have been utilized. Genetic Algorithm was utilized for learning the heaviness of every classifier. Advancement calculations are not considered for decreasing FAR.Methods used in Proposed System

\section{A. Image Acquisition}

Iris imaging a good ways off for an optical framework configuration is basic. In this manner for iris picture procurement, the focal point dependent on the geometry optics and the required parameters of camera are determined. Let the tallness of individual is $\mathrm{H}$, the computerized sensor's width as $\mathrm{W}$ and focal point of focal point are shown by $\mathrm{F}$, the item picture proportion as $\mathrm{M}$ and sensor pixel size as $\mathrm{S}$.

The distance D of an objected can be calculated from equation 1.

$$
D=F \frac{M}{S}
$$

The equation 2 shows the field depth in which the capturing volume is $\mathrm{V} \times \mathrm{A}$. 


$$
\begin{aligned}
& V=M W \\
& A=M H \\
& B=2 F \frac{(M-S)(M-S)}{S}
\end{aligned}
$$

By thinking about the above geometry optics, the focal point and the camera utilized in this article with casing pace of 30 edges for every second and the pixel size is 4-super pixels.

This camera can snaps a photo of human face totally with high-goals. The focal points with its opening size $\mathrm{F}$ is 15 and central length $300 \mathrm{~mm}$. So it can catch the iris picture unmistakably up to separation $4 \mathrm{~m}$ to $6 \mathrm{~m}$. Figure 2 shows distinctive iris acknowledgment frameworks models short proximity and long-go.

UBIRIS.v1 are the iris pictures which are caught from a good ways from 3 meters away by effectively looking through iris, face or palmprint designs. In this way in this article, the UBIRIS.V1 databases are utilized for the iris acknowledgment. The way toward catching the human face picture is appeared in figure 3 . In figure 4 the way toward removing the eye from the face is available.

\section{B. Processing of an Image}

The nature of iris picture got from the camera is poor. This can be occur because of the movement of individual, separation, obscure or impediments. In this manner it is important to pre-process the iris picture to improve the various parameters an iris picture, for example, differentiate, force, sign to commotion proportion, and so on for further preparing. The procedure which is utilized to improve the iris picture parameters are differentiation extending and histogram leveling. It just improves the nature of picture, it doesn't builds the data substance of a picture. The power scope of an iris picture is standardized to [0 1]. The sign to commotion proportion of iris picture is improved with the assistance of anisotropic dissemination channel. The device which is utilized for this reason for existing is MatLab understand the numerous brilliance changes. To locate the rank-request data and spatial data of an iris picture a weighted middle (WM) channel is utilized and this is one of the sorts of middle channel. The clamors shot and drive commotion are dismissed by the middle channel.

\section{Region extraction using Hough transform}

The boundary of the inner pupil and outer pupil of an iris picture can be recognized by utilizing the condition,

where, $\left(x_{0}, y_{0}\right)$ denotes the coordinates of a circle with radius $r$. The results obtained from Hough transform shows the boundary of pupil, eyelid extraction of iris image and the center of pupil.

The region of extraction is performed from the pre-processed image to perform the feature extraction effectively. HT [22] [23] is utilized for detecting the lines, circles, or any other parametric curves in the images and it enables the robust detection under noise and partial occlusion.

$$
\left(x-x_{0}\right)^{2}+\left(y-y_{0}\right)^{2}=r^{2}
$$

\section{Segmentation}

In a segmentation step the internal and external limits of the iris locale are recognized. This can accomplished by Hough transform. The Hough transform discovers various shapes like as circles or ovals of a picture. It is the initial phase in the Iris acknowledgment framework additionally the foundation of the total acknowledgment framework. It intends to recognize format, focuses, eyelids, eyelashes and radii, of the two iris fringe. Finding the lower and upper eyelid additionally separate eyelashes. With the assistance of the Daugman's Rubber Sheet Model the picture division, securing and highlight encoding of an iris picture can happens. It is additionally used to improve the nature of edges introduces in the picture. The most regularly utilized circle locator is integro-differential operator and it is numerically communicated as.

$M A X / G(r) * \frac{d}{d r} \int \frac{I(x, y)}{2 \pi r}$

where I ( $\mathrm{x}, \mathrm{y}$ ) means the input iris picture, $\mathrm{G}(\mathrm{r})$ indicates Gaussian with a standard deviation, $r$ is the sweep of round circular segment. It is the convolution activity which is appeared in image *. The line an iris picture can be recognized by the equation

$\mathrm{r}=\mathrm{x} \cos \theta+\mathrm{y} \sin \theta$

Where, $r$ is quantized separation and $\theta$ is quantized point. The $r$ and $\theta$ are thinking about quantized qualities in the pair $(\mathrm{r}, \theta)$.

\section{E. Normalization}

The Daugman's rubber sheet is a direct model that is allotted to the iris of the individual pixel dependent on the expansion, size and the genuine directions $(\mathrm{x}, \theta)$, where $\mathrm{x}$ is the unit interim and $\theta$ ranges from 0 and $2 \pi$. In this the segmented iris image is normalized into the block with equal in size respect to the block width $x$ and angular displacement $\theta$. The iris picture is remapped into the polar facilitate $(\mathrm{x}, \theta)$ framework from Cartesian composed (r, s) framework. Along these lines the standardized polar directions are $(\mathrm{x}, \theta)$ and the ordinary directions are (r, s).

Let the iris coordinates and iris boundaries of the pupil are represented as $\left(\mathrm{r}_{\mathrm{b}}, \mathrm{s}_{\mathrm{b}}\right)$ and $\left(\mathrm{r}_{\mathrm{e}}, \mathrm{s}_{\mathrm{e}}\right)$ along $\theta$ direction. The coefficient of an iris image won't be shifted regardless of whether the signal is distorted because of the camera and people position.

\section{F. Extraction of the segment features using ScatT-LOOP and LGP.}

The standardized iris picture is is fed to feature extraction, in which the highlights are extricated utilizing ScatT-LOOP descriptor, which is the integrated of LOOP [30], ST [31] and TT [29]. The ScatT-LOOP intends to get the surface highlights for precise iris acknowledgment to remarkably recognize the clients. In feature extraction step, the iris picture is grouped into new vessels picture. The standardized iris picture is exposed to perform the feature extraction by utilizing ScatT-circle descriptor.

Entelligence Engeering \& Sciences Publication 
The ScatT-circle produces the surface highlights for exact iris acknowledgment to remarkably distinguish the people. In this, the picture locale contains numerous vessel sections that are firmly separated with different directions and have a curved in nature. So for the estimation of highlight qualities, the new vessel sections are created from a double vessel maps. So as to discover nearby includes, a sub window of size $4 \times 4$ is made. The iris picture are analyzed through this sub window. Also, for each

sub window the quantity of vessel pixels and pixel enthusiasm can determined. The Kirsch veil is intended for the future extraction. The circle an incentive for the relating pixel is spoken to as,

$$
\operatorname{LOOP}\left(r^{*}, s^{*}\right)=\sum_{k=0}^{7} h\left(G^{k}-G^{*}\right) \cdot 2^{v}
$$

Where,

$$
h(d)=\left\{\begin{array}{lll}
1 & ; & \text { if } d \geq 0 \\
0 & ; & \text { Otherwise }
\end{array}\right.
$$

$\left(r^{*}, s^{*}\right)$ is the centre of the intensity of iris image. $\mathrm{G}_{\mathrm{k}}$ is the neighborhood pixel intensity, $\mathrm{G}$ is the original image pixel intensity, and $k$ takes the value ranges from 0 to 7 .

\section{G. Tetrolet Transform}

The tetrolet descriptor [29] is used for supporting tetrominoes, which is framed by getting four squares together with comparable size. Here, the low-pass info picture is separated into squares and nearby tetrolet premise are acquired dependent on the geometry of the picture

a) Initialization: The segmented input image is split into blocks of size $4 \times 4$.

b) Representation of image blocks as the sparsest tetrolet: Each of the image blocks is subjected to the sparsest tetrolet representation and for every individual blocks, a total of 117 tetromino coverings are admitted each of which is given to the Haar wavelet transform along with four low pass coefficients for generating 12 coefficients. For the individual block, the tetrolet decomposition is done at the optimum based on 12 tetrolet coefficients to determine the final sparse image.

c) Representation of the high pass and Low pass coefficients: The steps involved in the Tetrolet decomposition algorithm is preceded with the arrangement of $2 \times 2$ matrix based on reshape function.

d) Tetrolet Coefficients: After representing the sparse matrix for the individual blocks, the high pass, as well as the low pass matrices, is kept safe for the use of the future.

e) Termination: The steps (b) to (c) are repeated for the low pass image and the output obtained is the binary image, which is denoted as, $\mathrm{D}_{2}$.

\section{H. Scattering transform}

ST [31] is used for producing texture highlights for the picture $\mathrm{D}_{2}$ to render exceptionally strong highlights and a locally invariant component that are made dependent on Morlet wavelet with direction and various scales. The steps involved in the ST are depicted underneath:

f) Transformation of Local affine to preserve the image: The Local affine transformation is completed utilizing convolution of individual picture sections with low pass channel, which works dependent on the scaling factor for forestalling the twisting in the info picture. The high- frequency segments are dispensed with dependent on nearby relative transformation.

\section{g) Capturing high-frequency components based on} Morlet filters: The high-frequency parts are acquired dependent on wavelet coefficients, which is the consequence of Morlet or bandpass and the normal filters.

h) Obtain the scattering coefficients: The wavelet modulus transformation is used for creating the scattering coefficients. The outcome acquired from ST is represented to as, $\mathrm{D}_{4}$

\section{Extraction of the features using LGP}

LGP [33] is the human and face portrayal approach that is regardless to the nearby force varieties with the edges for producing the steady designs. In the event that the slope estimation of the neighboring pixel is higher than the edge the threshold value, at that point the value assigned to the pixel as 1 or the worth is 0 . Let us assume a circle with a radius $r$ centered on the pixel by taking $n$ sample points on the circle. LGP employs the bilinear interpolation for computing the neighborhood pixel values for $r$ and $n$. It uses $|2 \times r \times 1|$ by $|2 \times r \times 1|$ the kernel to summarize the local structure of the iris. At the center pixel position (x,y), LGP takes $|2 \times r \times 1|$ by $|2 \times r \times 1|$ neighboring pixels. The gradient value between the neighboring pixel $\mathrm{G}_{0}$ and the center pixel $G_{Z}$ as, $Y_{0}=\left|G_{0}-G_{Z}\right|$ and the average gradient value of $r$ is represented as,

$$
\bar{y}=\frac{1}{r} \sum_{o r=0}^{r-1} y_{o}
$$

Thus, LGP $(\mathrm{x}, \mathrm{y})$ is represented as,

$$
\operatorname{LGP}(x, y)=\sum_{r=0}^{r-1} s\left(y_{o}-\bar{y}\right) 2^{o}
$$

$$
\text { where, } s(r)= \begin{cases}0, & \text { if } r<0, \\ 1, & \text { otherwise. }\end{cases}
$$

Feature vector: The feature vector is represented as,

$$
\delta=\left\{\delta_{1}, \delta_{2}, \ldots, \delta_{w}\right\} ; \text { for }(1<\tau<w)
$$

where, the dimension of the input image is represented as $\{1 \times W\}$, and $W$ is the total feature dimension.

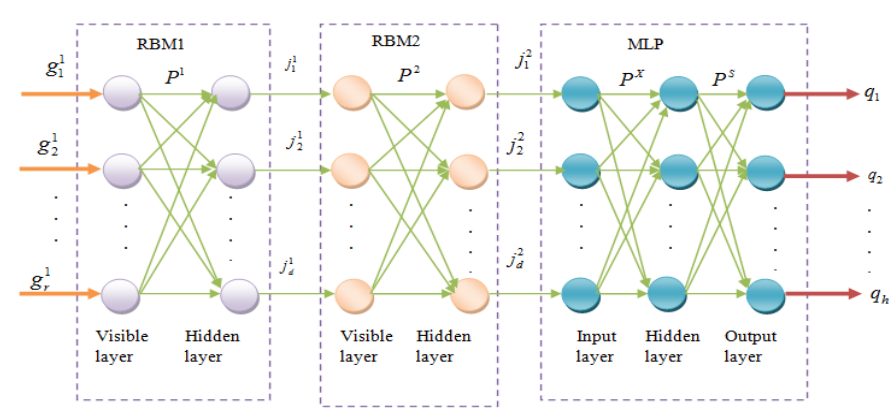

Fig. 2. Architecture of DBN classifier

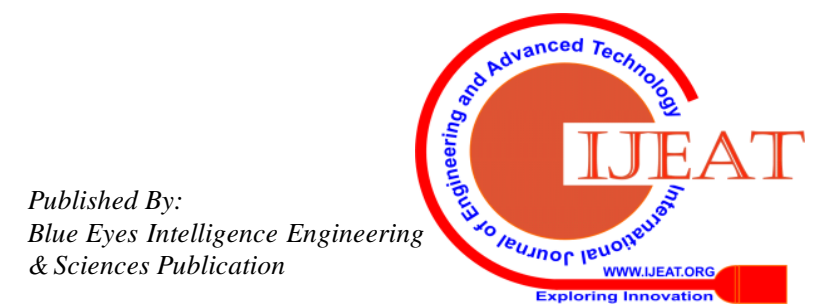


Iris Recognition using the Deep Belief Neural $\operatorname{Network}(D B N)$

This section deals with the employed steepest gradient-based DBN for effective iris recognition.

The features obtained using ScaTLOOP and LGP descriptor are given to the DBN classifier for iris recognition.

After the extraction of features, iris recognition is performed using DBN. The proposed DBN classifier is designed using a single MLP layer and two RBM layers, as shown in figure 1. The input for the first RBM in DBN is the feature vector of size, $(n \times z)$. The result obtained from the hidden layer forms the input for the second RBM, and its output is the input to the MLP.

The DBN classifier utilizing the Chronological MBO calculation is utilized for the recognizable proof of an individual. The non-linear complex connection introduces in the genuine are expelled by utilizing DBN classifier and the sequential MBO calculation prepares the DBN classifier. The iris acknowledgment is performed utilizing the sequential MBO-based DBN, which is the mix of the ordered idea with the MBO calculation to prepare DBN that relies upon the movement highlights of the monarch butterfly. The standard MBO fuses the tweaking of parameters and the unpredictable free calculation to upgrade the presentation of the proposed sequential MBO-based DBN, and the high dimensional issues are adequately managed utilizing MBO. Figure 1 demonstrates the Architecture of DBN classifier.

In any case, the looking through speed and the combination speed are upgraded by coordinating the sequential idea, which characterizes the arrangements (inclinations and loads) from the previous cycles to amend the new biases and weights.

The visible layer, which has the feature vector as its input, and the hidden layer of RBM1 can be given as,

$$
\begin{aligned}
& g^{1}=\left\{g_{1}^{1}, g_{2}^{1}, \ldots, g_{q}^{1}, \ldots, g_{z}^{1}\right\} ; 1 \leq q \leq z \\
& j^{1}=\left\{j_{1}^{1}, j_{2}^{1}, \ldots, j_{n}^{1}, \ldots, j_{r}^{1}\right\} ; 1 \leq n \leq r
\end{aligned}
$$

where, $g_{q}^{1}$ indicates the $\mathrm{q}^{\text {th }}$ visible neuron in RBM1, $j_{r}^{1}$ denotes then $\mathrm{n}^{\text {th }}$ hidden neuron and $\mathrm{r}$ be the total number of hidden neurons. Each neuron in the hidden layer and the visible layer has a bias. Consider $\mathrm{p}$ and $\mathrm{q}$ as the biases in the hidden and the visible layer. The two biases belongs to the neurons in both the layers for the first RBM are given by,

$$
\begin{aligned}
& p^{1}=\left\{p_{1}^{1}, p_{2}^{1}, \ldots, p_{q}^{1}, \ldots, p_{z}^{1}\right\} \\
& q^{1}=\left\{q_{1}^{1}, q_{2}^{1}, \ldots, q_{n}^{1}, \ldots, q_{r}^{1}\right\}
\end{aligned}
$$

where, $p_{q}^{1}$ be the bias related with visible neuron and $q_{n}^{1}$ denotes the bias corresponding to hidden neuron. For the first RBM, the weight vector is expressed as,

$$
w^{1}=\left\{w_{q n}^{1}\right\} ; 1 \leq q \leq z ; 1 \leq l \leq r
$$

where, $w_{q n}^{1}$ represents the weight between $\mathrm{n}^{\text {th }}$ hidden neuron and $\mathrm{q}^{\text {th }}$ visible neuron and the weight vector size is zxr. Hence, the output of the hidden layer in RBM1 can be computed using the weights and bias corresponding to every visible neuron as,

$$
f_{n}^{1}=\sigma\left[b_{n}^{1}+\sum_{q} m_{q}^{1} w_{q n}^{1}\right]
$$

where, the activation function is denoted as, $\sigma$.

The total visible neurons are equivalent to the total number of hidden neurons in the first RBM and are represented as,

$$
m^{2}=\left\{m_{1}^{2}, m_{2}^{2}, \ldots, m_{p}^{2}\right\}=\left\{f_{n}^{1}\right\} ; 1 \leq n \leq p
$$

Similarly, hidden layers of remaining layer can be calculated.

\section{H. Training of Deep Belief Network}

The training process of the DBN classifier. RBM has unsupervised learning based on gradient descent method, whereas MLP performs supervised learning method using standard backpropagation approach. Therefore, DBN is trained based on gradient descent- backpropagation algorithm. Here, the most appropriate weights are chosen optimally for the update. The training procedure of the proposed DBN classifier is described below,

A training sample $\mathrm{N}$ is given as the input to the first layer of RBM. It computes the probability distribution of the data and encodes it into the weight parameters. The steps involved in the training process of RBM is illustrated as,

Step 1: At first, the input training sample is read and the weight vector $\mathrm{W}^{1}$ is produced in random as shown in equation (15)

Step 2: The probability function of each hidden neuron in the first RBM is calculated as,

$$
P\left(f_{n l}^{1}=1 \mid m^{1}\right)=\sigma\left[b_{n}^{1}+\sum_{q} m_{q}^{1} w_{q n}^{1}\right]
$$

Step3: The positive gradient $\theta^{+}$is computed using visible vector and the probability of the hidden layer as,

$$
\theta^{+}=m^{1} \cdot P_{f}^{1^{T}}
$$

where, the visible vector is denoted as $m^{1}$, and $P^{1^{T}}$ is the probability of hidden neurons.

Step 4: The probability of each visible neuron is obtained by sampling a reconstruction of the visible from the hidden layer as,

$$
P\left(m_{q}^{\prime 1}=1 \mid f^{1}\right)=\sigma\left[v_{q}^{1}+\sum_{n} f_{n}^{1} w_{q n}^{1}\right]
$$

Published By: 
where, $\mathrm{m}^{\prime}$ refer to the restoration of visible neurons and $v_{q}^{1}$ denotes the bias of visible unit in the layer of first RBM.

Step 5: Then, the probability of the reconstruction hidden neurons are obtained by resampling the hidden states from $\mathrm{m}^{\prime}$ as,

$$
P\left(f_{n}^{\prime 1}=1 \mid f m^{1}\right)=\sigma\left[q_{n}^{1}+\sum_{q} m_{q}^{\prime 1} w_{q n}^{1}\right]
$$

Where, the reconstruction of hidden neurons is denoted as $\mathrm{f}^{\prime}$.

Step 6: The negative gradient using $\mathrm{f}^{1}$ and $\mathrm{m}$ 'l are computed as,

$$
\theta^{-}=m^{\prime 1} \cdot f^{1^{T}}
$$

Step 7: Weights are updated by subtracting the negative gradient from the positive gradient, as given as,

$$
\Delta w_{q n}=\eta\left(\theta^{+}-\theta^{-}\right)
$$

where, the learning rate is denoted as $\prod$.

Step 8: Update the weights for the next iteration $\mathrm{t}+1$ based on steepest or gradient descent algorithm as,

$$
w_{q n}^{1}(t+1)=w_{q n}^{1}(t)+\Delta W_{q n}
$$

Where, the weight at current iteration is denoted as $w_{q n}^{1}$.

Step 9: Calculate the energy for a joint configuration of the neurons in the visible and the hidden layers as,

$$
G^{B}\left(m^{1}, f^{1}\right)=-\sum_{q, n} w_{q n}^{1} m_{q}^{1} f_{n}^{1}-\sum_{q} v_{q}^{1} m_{q}^{1}-\sum_{n} b_{n}^{1} f_{n}^{1}
$$

where, $w_{q n}^{1}$ is the weight of the first RBM layer.

Step 10: Finally, the weights providing minimum energy is chosen as the optimal weight.

The output attained from RBM layer 1 is passed to the RBM2 visible layer and determines the probability distribution in DBN. The energy is computed for the weights $w^{2}$ and the weight with less energy is selected for the next iteration.

\section{Performance metrics}

The metrics used to evaluate the methods, are FRR, FAR, and accuracy are explained below.

1) Accuracy: The accuracy measures the accurateness of iris recognition based on iris modality and is represented as,

$$
\text { Accuracy }=\frac{J c+J u}{J c+R c+J u+R u}
$$

where, $J u$ denotes the true positives, and $J c$ is the true negatives. $R u$ denotes the false positives and $R c$ is the false negatives.
2) Sensitivity: Sensitivity is otherwise called True positive Rate (TPR), which is the measure of positive-ness identified correctly, and is calculated using the below equation.

Sensitivity $=\frac{J u}{R c+J u}$

3) False Rejection Ratio (FRR): FRR is the ratio of false rejection to the genuine attempts, and is expressed as,

$$
F R R=\frac{R c}{J u+R c}=1-T P R
$$

4) Specificity: Specificity or True Negative Rate (TNR) is the measure of false negatives, which are correctly located. Specificity is expressed as,

Specificity $=\frac{J c}{J c+R u}$

5) False Acceptance Rate (FAR): FAR is the ratio of false attempt to imposter attempts, and is represented as,

$$
F A R=\frac{R u}{R u+J c}=1-T N R
$$

6) Receiver Operating Characteristics (ROC): ROC refers to the relationship between TNR and TPR, which is used to compute the performance of the system.

\section{Building Block Diagram Of Projected STRUCTURE}

The implementation of this work is given in this step. Figure 2 shows suggest method for iris recognition. Here during this unit diagram of prompt structure is given.

\section{Images Which are Test}

The database utilized for this object is UBIRIS.V1. The total database of this segment was searched for Iris pictures. Here there are 20 people groups dataset is to be considered for the examination.

\section{Pre-processing and De-noising}

Iris Recognition a ways off (IAAD) is utilized to improve the differentiation of the picture. Middle based channels are utilized to evacuate the sound introduces in the debased iris pictures.

In pre-handling, the sign to-commotion portion of iris picture profile is upgraded additionally for this goal.

\section{Hough Transform}

The Hough transform is used to extracts the different curves or shapes of an iris image.

\section{Segmentation and Normalization}

The division of the districts can occur by considering comparable properties of an iris picture. A portion of the comparable properties are shading, brilliance, differentiate, surface, dark level, and so forth. In this division step, the iris picture is part into disparate outskirts.

Published By: 
The portioned iris picture is set up by utilizing a standardization calculation. The fragmented iris picture is utilized for the future extraction

process. Because of the shifting position of an individual and the camera, the iris picture is exceptionally influenced by mutilation.

\section{Feature Extraction}

The major plan of the BPNN presents 1 info layer and least 1 shrouded layer pursued by yield layer.

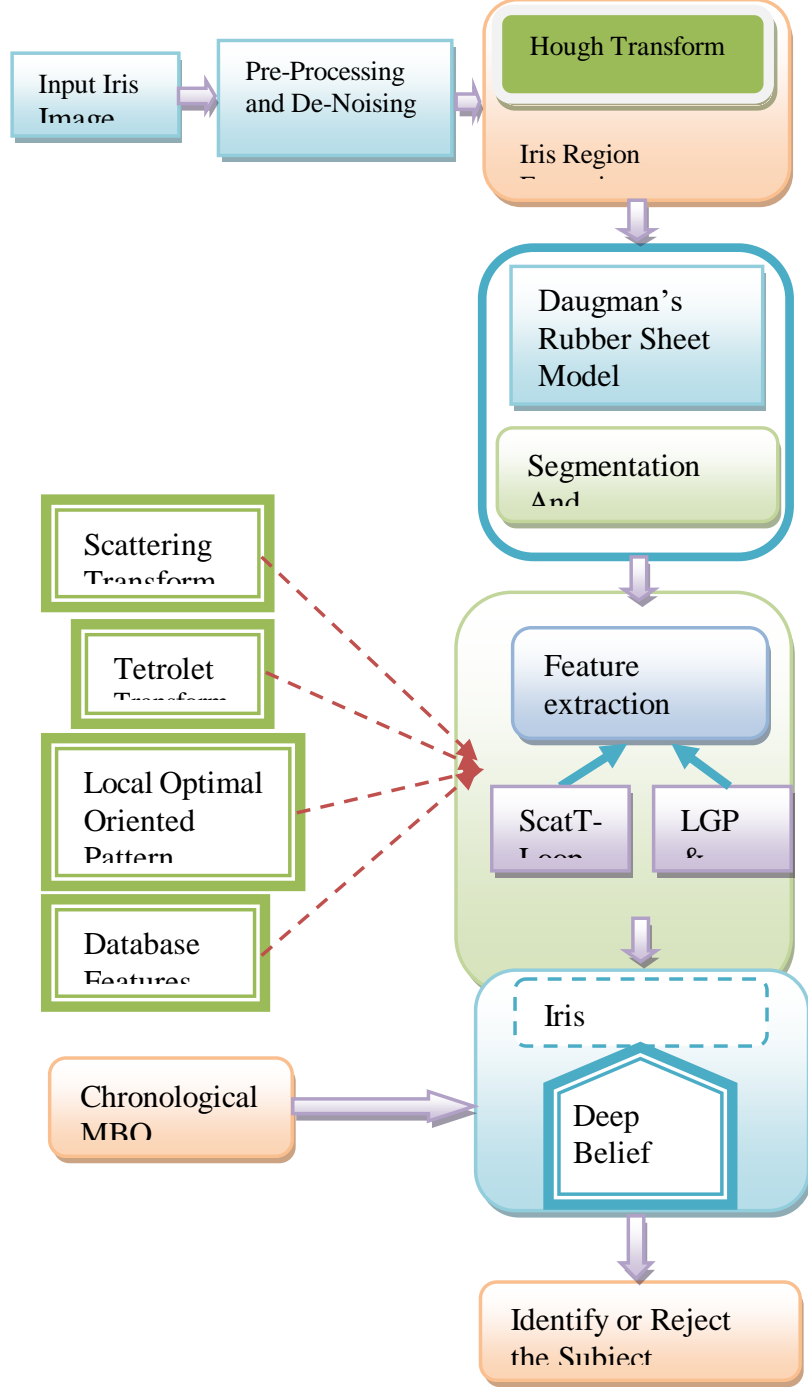

Fig. 3.Suggest Method For Iris Recognition.

For preparing multi-layer artificial neural system Back Propagation Neural Network (BPNN) is normally utilized. PCA is an ideal plan to pack the high dimensional vectors into the low dimensional vectors and register the parameters from the information legitimately. The ScatT-Loop generator is use to remove the various highlights of an iris image. The ScatT-Loop creates the surface highlights for precise iris acknowledgment to remarkably recognize the people. Administrator in LGP uses the angle pixel esteems and is resolved as the force esteem. Distinctive changes shows in this progression are utilized to compute the surface highlights for the iris picture.

\section{Deep Belief Network (DBN)}

The powerful acknowledgment is acquired by the ideal tuning of the DBN classifier utilizing the Chronological MBO calculation. By utilizing Chronological MBO calculation we can gauge or characterize exact Iris picture. The dimensionally decreased highlights got utilizing PCA is bolstered as contribution to DBN to perceive the people.

\section{EXPERIMENTAL RESULTS}

The results achieved by the proposed iris recognition based on DBN are given in this section. The step by step execution of the proposed system is presented. Firstly we take input test iris image. In this paper, there are 20 peoples dataset is to be considered for the experiment. The database used for this purpose is UBIRIS.v1[26]. Figure 4 shows the key in iris recognition image having the dimension 512 x 512. This image can be selected by using the GUI in MatLab. Figure 4 shows the input images and the pre-processed images employed for iris recognition. Figure 5 depicts the output of Hough Transform to the input images 1 and 2 .

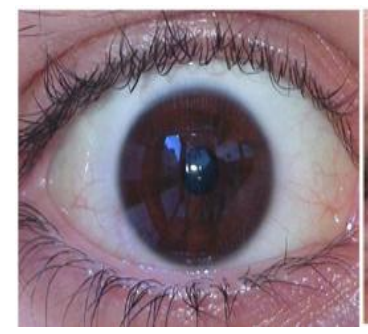

a)

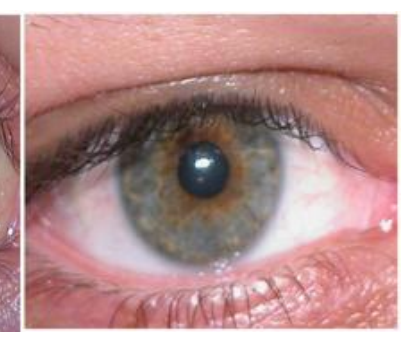

b)
Fig. 4. Sample results of Input Images.

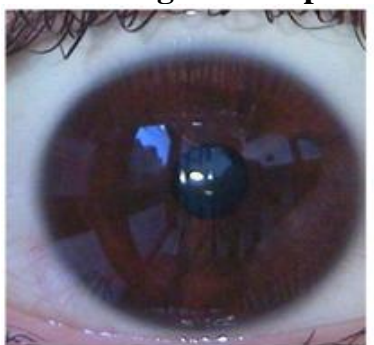

a)

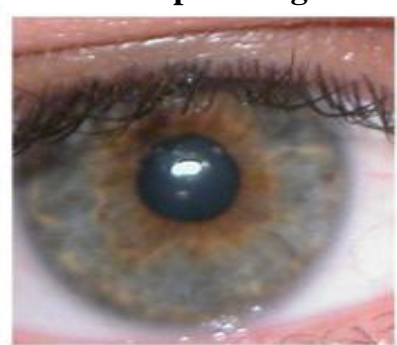

b)
Fig. 5.Hough Transform of Input Iris a) Image 1 and b) Image 2.

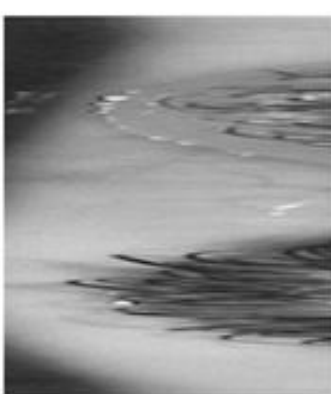

a)

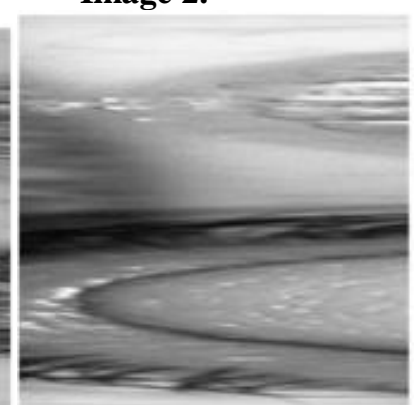

b)
Fig. 6.Suggest Daugman's rubber sheet model for a) Input image 1 and b) Image 2. 


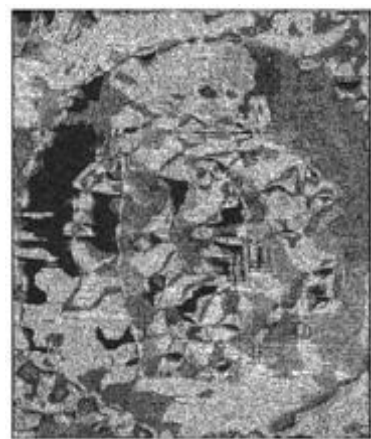

a)

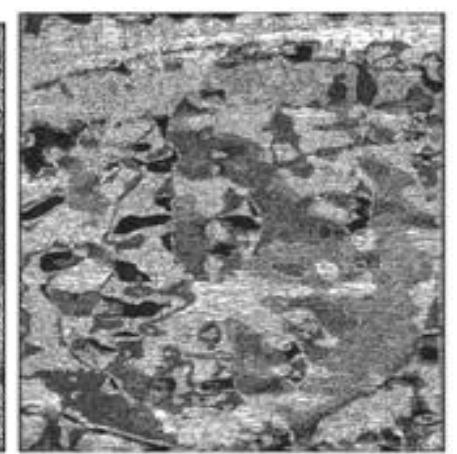

b)
Fig. 7.Suggest Output of ScatT-LOOP corresponding to for a) Input image 1 and b) Image 2.

Figure 6 shows the suggest Daugman's rubber sheet model for a) Input image 1 and b) Image 2 .

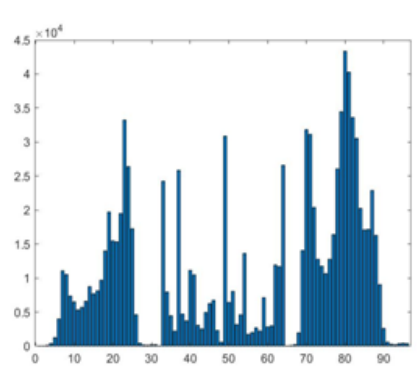

a)

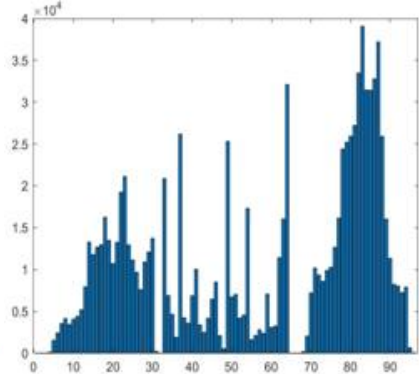

b)
Fig. 8.Suggest Histogram corresponding to a) Input image 1 and b) Image 2.

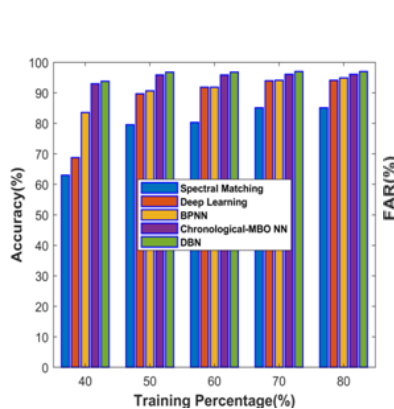

a)

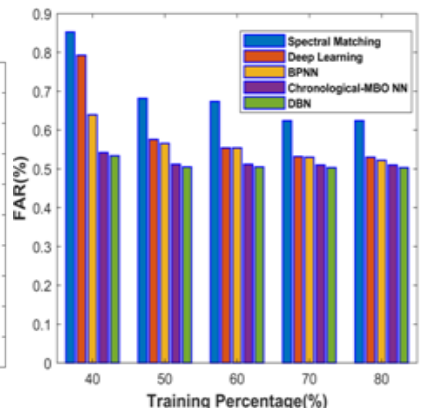

b)
Fig. 9.Suggest Comparative analysis by varying the training percentage using Image 1 a)Accuracy, b) FAR.

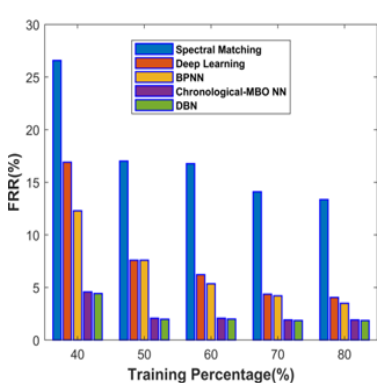

a)

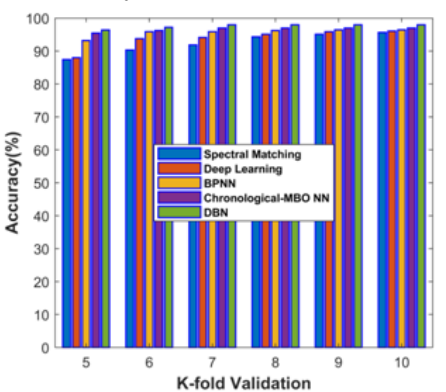

b)
Fig. 10. Suggest Comparative analysis by varying the training percentage using Image 1 a) FRR, b) $\mathrm{K}$ fold accuracy.

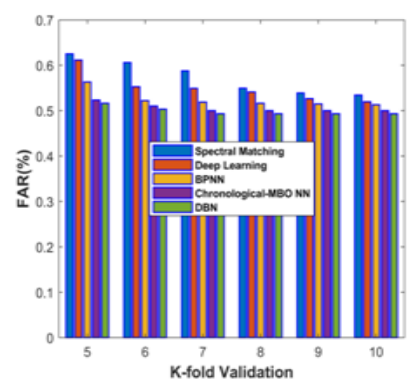

a)

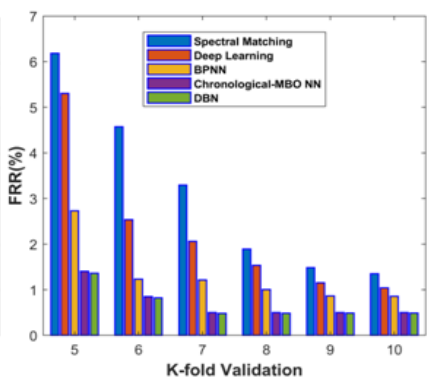

b)
Fig. 11. Suggest Method For Comparative analysis by varying the K-Fold Image 1 b) FAR c) FRR.

Figure 7 shows the normalized output images by using ScatT-LOOP for a) Input image 1 and b) Image 2. Figure 8 shows the Histogram corresponding to a) Input image 1 and b) Image 2. Figure 9 suggest comparative analysis by varying the training percentage using Image 1Accuracy, b) FAR.

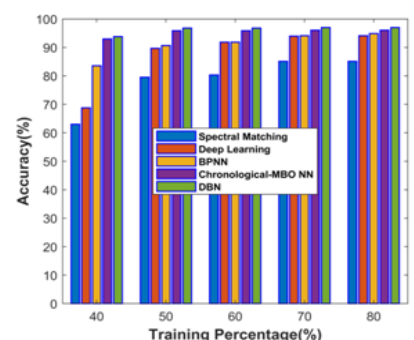

a)

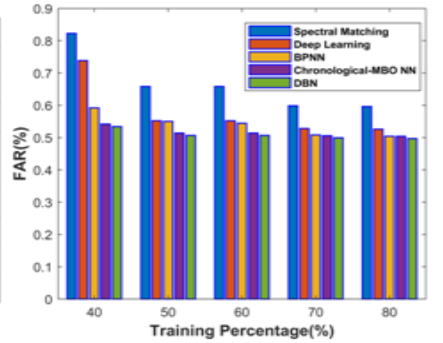

b)
Fig. 12. Suggest Comparative analysis by varying the training percentage using Image 2 a)Accuracy, b)

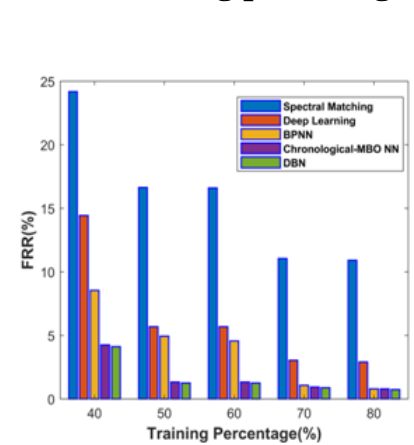

a)

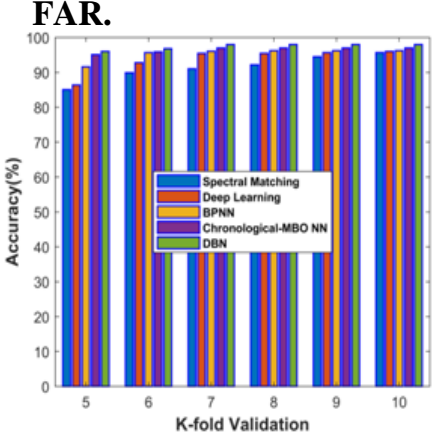

b)
Fig. 13. Suggest Comparative analysis by varying the training percentage using Image 2 a) FRR, b) Kfold accuracy.

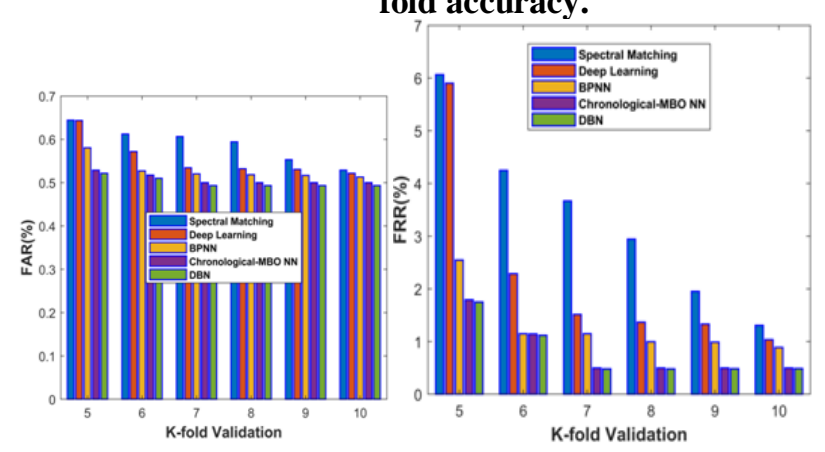

a)

b)

Fig. 14. Suggest Method For Comparative analysis by varying the K-Fold Image 2 b) FAR c) FRR.

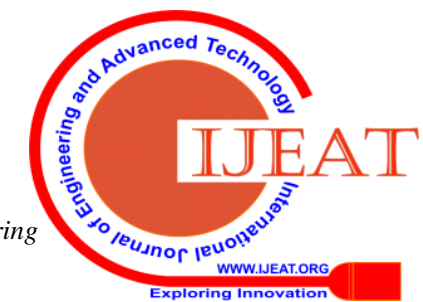




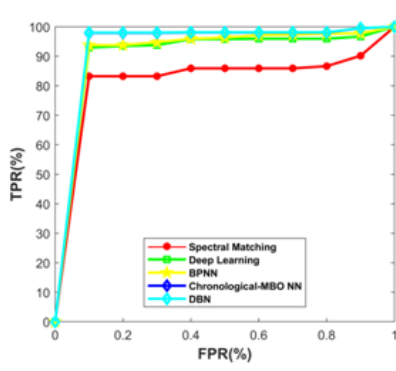

a)

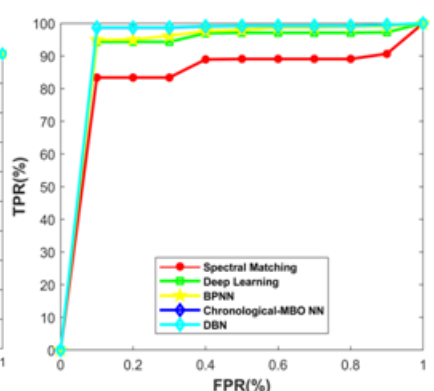

b)
Fig. 15. Suggest Method Analysis of ROC a) Using image 1, b) using Image 2 For Iris Recognition.

Figure 10 Suggest Comparative analysis by varying the training percentage using Image 1 a) FRR, b) K- fold accuracy. Figure 11 Suggest Method For Comparative analysis by varying the K-Fold Image 1 b) FAR c) FRR. Figure 12 Suggest Comparative analysis by varying the training percentage using Image 2 a) Accuracy, b) FAR. Figure 13 Suggest Comparative analysis by varying the training percentage using Image 2 a) FRR, b) K- fold accuracy. Figure 14 Suggest Method For Comparative analysis by varying the K-Fold Image 2 b) FAR c) FRR. Figure 15 Suggest Method Analysis of ROC a) Using image 1, b) using Image 2 For Iris Recognition.

TABLE I. COMPARATIVE ANALYSIS BASED ON TRAINING PERCENTAGE

\begin{tabular}{|c|c|c|c|}
\hline Methods & Accuracy (\%) & FAR (\%) & FRR (\%) \\
\hline Spectral matching & 85.09 & 0.6238 & 13.35 \\
\hline Deep learning & 94.11 & 0.53 & 4.05 \\
\hline BPNN & 94.88 & 0.522 & 3.5 \\
\hline $\begin{array}{c}\text { Chronological } \\
\text { MBO-NN }\end{array}$ & 96.03 & 0.51 & 1.928 \\
\hline \begin{tabular}{c} 
Proposed DBN \\
\hline
\end{tabular} & 96.979 & 0.503 & 1.864 \\
\hline
\end{tabular}

Table 1 shows the comparative analysis based on training percentage of Spectral matching, Deep learning, BPNN, Chronological MBO-NN and Proposed DBN for Accuracy, FAR and FRR.

\begin{tabular}{|c|c|c|c|}
\hline Methods & Accuracy (\%) & FAR (\%) & FRR (\%) \\
\hline Spectral matching & 95.65 & 0.534 & 1.34 \\
\hline Deep learning & 96.04 & 0.519 & 1.03 \\
\hline BPNN & 96.42 & 0.513 & 0.85 \\
\hline $\begin{array}{c}\text { Chronological } \\
\text { MBO-NN }\end{array}$ & 97 & 0.5 & 0.5 \\
\hline Proposed DBN & 97.96 & 0.493 & 0.48 \\
\hline
\end{tabular}

Table 2 shows the comparative analysis based on K-fold of Spectral matching, Deep learning, BPNN, Chronological MBO-NN and Proposed DBN for Accuracy, FAR and FRR.

\section{Conclusion}

This paper introduces the DBN classifier for iris acknowledgment for which at first, the iris picture is pre-handled, and the iris area is removed utilizing HT. From that point onward, the division and the standardization of the iris are performed based the Daugman's rubber sheet model stays as the promoter of the acknowledgment precision. At that point, the outcome got from the division is given to the element extraction module in which the highlights are extricated utilizing ScaT-LOOP, which is the incorporation of ST, TT, and LOOP descriptor and LGP descriptor. At last, steepest slope based DBN is used for powerful iris acknowledgment. In addition, the presentation of the proposed strategy is estimated dependent on measurements, for example, precision, FAR, and FRR through shifting the preparation information rate and K-Fold utilizing UBIRIS.v1 iris dataset. The aftereffects of the DBN classifier are thought about against the current systems by accomplishing greatest precision estimation of $97.9 \%$, the base FAR estimation of $0.493 \%$, and the base FRR estimation of $0.48 \%$ that demonstrates its predominance. The future component of the exploration will be focused on broadening the investigation utilizing other standard databases with profoundly propelled highlights.

\section{ACKNOWLEDGMENT}

The authors want to thank the Bharath Institute of Higher Education and Research, Bharath University,Selaiyur, Chennai, CSE Department, R \& D Bharath University.Also thanks to STE's NBN Sinhgad School of engineering, Ambegaon(Bk),Pune.

\section{REFERENCES}

[1] Randa F. Soliman, Mohamed Amin and Fathi E. Abd El-Samie, "A Novel Cancelable Iris Recognition Approach novel cancelable iris recognition system based on feature learning techniques", Information Sciences, vol.406, pp.102-118, 2017.

[2] Shubhika Ranjan, S.Prabu, Swarnalatha P, Magesh G, Ravee Sundararajan," Iris recognition system", International Research Journal of Engineering and Technology (IRJET) vol. 4, no.: 12, pp.2395-0056, December 2017.

[3] Xiaonan Liu, Yuchen Bai, Yuwen Luo, Zhengwei Yang, and Yan Liu, "Iris recognition in visible spectrum based on multi-layer analogous convolution and collaborative representation", Pattern Recognition Letters, vol. 117, pp. 66-73, 2019.

[4] Heinz Hofbauer, Ehsaneddin Jalilian, and Andreas Uhl, "Exploiting superior CNN-based iris segmentation for better recognition accuracy", Pattern Recognition Letters, vol.120, pp.17-23, 2019.

[5] Kevin W. Bowyer, Karen Hollingsworth, and Patrick J. Flynn, "Image understanding for iris biometrics: A survey", Computer Vision and Image Understanding, vol. 110, pp. 281-307, 2008.

[6] John Daugman," How iris recognition works", The essential guide to image processing, pp.715-739, 2009.

[7] A. Alice Nithya and C. Lakshmi," Enhancing iris recognition framework using feature selection and BPNN", Cluster Computing, pp:1-10, 2018.

[8] Richa Gupta and Priti Sehgal," A Complete End-to-End System for Iris Recognition to Mitigate Replay and Template Attack", In Soft Computing and Signal Processing, pp. 571-582, 2019.

[9] Milad Salem, Shayan Taheri and Jiann-Shiun Yuan," Utilizing Transfer Learning and Homomorphic Encryption in a Privacy Preserving and Secure Biometric Recognition System. Computers, vol.8, no.1, pp.3, 2019.

[10] Maryam Mostafa Salah, Sameh A. Napoleon, El-Sayed M. El-Rabaie, Fathi E. Abd El-Samie, and Mustafa M. Abd Elnaby, "Sensitivity Analysis of a Class of Iris Localization Algorithms to Blurring Effect",Wireless Personal Communications, vol. 104, no.1,pp.269-286.

[11] Caiyong Wang, Yuhao Zhu, Yunfan Liu, Ran He, and Zhenan Sun, "Joint Iris Segmentation and Localization Using Deep Multi-task Learning Framework", 2019. 
[12] Fernando Alonso-fernandez, Reuben A. Farrugia, Josef Bigun, Julian Fierrez, and Ester Gonzalez-Sosa", A Survey of Super-Resolution in Iris Biometrics with Evaluation of Dictionary-Learning", IEEE Access, vol.7, pp.6519-6544, 2019.

[13] Ishan Nigam, Mayank Vatsa, Richa Singh, "Ocular biometrics: A survey of modalities and fusion approaches", Information Fusion, vol.26, pp.1-35, 2015

[14] A. Alice Nithya, and C. Lakshmi, "On the performance improvement of non-cooperativeiris biometrics using segmentation and feature selection techniques",International Journal of Biometrics, vol.11, no.1, pp.1-21, 2019.

[15] S. G. Gino Sophia, and V. Ceronmani Sharmila, "Morphological-Based Localization of an Iris Image", In proceedings of First International Conference on Artificial Intelligence and Cognitive Computing, pp. 27-38, 2019.

[16] Jus Lozej, Dejan Stepecy, Vitomir Strucz, and Peter Peer, "Influence of segmentation on deep iris recognition performance", 2019.

[17] Chung-Chih Tsai, Heng-Yi Lin, Jinshiuh Taur, and Chin-Wang Tao, "Iris Recognition Using Possibilistic Fuzzy Matching on Local Features", IEEE transactions on systems, man, and cybernetics - part b: cybernetics, vol. 42, no. 1, February 2012.

[18] Maha Sharkas, "A Neural Network Based Approach for Iris Recognition Based on Both Eyes", In proceedings of international conference on SAI Computing, July 2016.

[19] N. Pattabhi Ramaiah, Ajay Kumar, "Towards More Accurate Iris Recognition using Cross-Spectral Matching", IEEE transactions on Image processing, vol.26, no.1, pp.208-221.

[20] Chun-Wei Tan and Ajay Kumar,"Efficient and Accurate At-a-Distance Iris Recognition Using Geometric Key-Based Iris Encoding", IEEE transactions on information forensics and security, vol. 9, no. 9, September 2014.

[21] Kien Nguyen, Clinton Fookes, Arun Ross, and Sridha Sridharan, "Iris Recognition with Off-the-Shelf CNN Features: A Deep Learning Perspective", IEEE access, September 2017.

[22] Neda Ahmadi and Gholamreza Akbarizadeh, "Hybrid robust iris recognition approach using iris image pre-processing, two-dimensional gabor features and multi-layer perceptron neural network/PSO", IET Biometrics, vol.7, no.2, pp.153-162, 2017.

[23] Kiran B. Raja, R. Raghavendra, Sushma Venkatesh, and Christoph Busch., "Multi-patch deep sparse histograms for iris recognition in visible spectrum using collaborative subspace for robust verification", Pattern Recognition Letters, vol.91, pp.27-36, 2017.

[24] Soubhagya Sankar Barpanda, Pankaj K. Sa, Oge Marques, Banshidhar Majhi, Sambit Bakshi, "Iris recognition with tunable filter bank based feature", Multimedia Tools and Applications, vol.77, no.6, pp.7637-7674, 2018.

[25] Abhishek Gangwar, and Akanksha Joshi, "DeepIrisNet: Deep Iris Representation with Applications In Iris Recognition And Cross-Sensor Iris Recognition", In proceedings of IEEE International Conference on Image Processing (ICIP), pp. 2301-2305, 2016.

[26] Ashok K Bhateja, Shikhar Sharma, Santanu Chaudhury, and Nitin Agrawal,"Iris Recognition based on Sparse Representation and k-nearest subspace with Genetic Algorithm", Pattern Recognition Letters, vol.73, pp.13-18, 2016.

[27] Kamal Hajari, and Kishor Bhoyar,"A Review of Issues and Challenges in Designing Iris Recognition Systems for Noisy Imaging Environment",In proceedings of International Conference on Pervasive Computing (ICPC), pp. 1-6, 2015.

[28] Wenbo Dong, Zhenan Sun, and Tieniu Tan," A design of iris recognition system at a distance",In proceedings of Chinese Conference on Pattern Recognition, pp. 1-5, 2009.

[29] Jens Krommweh, "Tetrolet Transform: A New Adaptive Haar Wavelet Algorithm for Sparse Image Representation", Journal of Visual Communication and Image Representation, vol.21, no.4, pp.364-374, 2010 .

1. pp. 740-741 [Dig. $9^{\text {th }}$ Annu. Conf. Magnetics Japan, 1982, p. 301].

2. M. Young, The Techincal Writers Handbook. Mill Valley, CA University Science, 1989

3. (Basic Book/Monograph Online Sources) J. K. Author. (year, month, day). Title (edition) [Type of medium]. Volume(issue). Available: http://www.(URL)

4. J. Jones. (1991, May 10). Networks (2nd ed.) [Online]. Available: http://www.atm.com

5. (Journal Online Sources style) K. Author. (year, month). Title. Journal [Type of medium]. Volume(issue), paging if given. Available: http://www.(URL)
[30] Tapabrata Chakraborti, Brendan McCane, Steven Mills, and Umapada Pal,"LOOP Descriptor: Local Optimal Oriented Pattern", IEEE Signal Processing Letters, vol.25, no.5, pp.635-639, 2018.

[31] Prateekshit Pandey, Richa Singh, Mayank Vatsa, "Face Recognition using Scattering Wavelet under Illicit Drug Abuse Variations", In proceedings of International Conference on Biometrics (ICB), pp. 1-6), 2016.

[32] Alaa S. Al-Waisy, Rami Qahwaji, Stanley Ipson, Shumoos Al-Fahdawi and Tarek A. M. Nagem," A multi-biometric iris recognition system based on a deep learning approach", Pattern Analysis and Applications, vol :21,no: 3, pp :783-802, August 2018.

[33] B. Jun, I. Choi and D. Kim, "Local Transform Features and Hybridization for Accurate Face and Human Detection," in IEEE Transactions on Pattern Analysis and Machine Intelligence, vol. 35, no. 6, pp. 1423-1436, June 2013

[34] 200F. Juefei-Xu, M. Savvides, Unconstrained periocular biometric acquisition and recognition using COTS PTZ camera for uncooperative and non-cooperative subjects, in: IEEE Workshop on Applications of Computer Vision (WACV), 2012, pp. 201-208.

[35] 202 H.Proenca, J. Briceno , Periocular biometrics: constraining the elastic graph matching algorithm to biologically plausible distortions, IET Biom. 3 (2014) . 167-75

[36] 189C. Padole, H. Proenca, Compensating for pose and illumination in uncon- strained periocular biometrics, Int. J. Biom. 5 (2013). 336-59

[37] 198C. Padole, H. Proenca, Periocular recognition: analysis of performance degra- dation factors, in: International Conference on Biometrics (ICB), 2012, pp. 439-445.

[38] 194J. Adams, D. Woodard, G. Dozier, P. Miller, K. Bryant, G. Glenn, Genet- ic-based type ii feature extraction for periocular biometric recognition: less is more, in: International Conference on Pattern Recognition (ICPR), 2010, pp. 205-208.

[39] 192P. Miller, J. Lyle, S. Pundlik, D. Woodard, Performance evaluation of local ap- pearance based periocular recognition, in: IEEE International Conference on Biometrics: Theory Applications and Systems (BTAS) 2010a, pp. 1-6.

[40] 191D. Woodard, S. Pundlik, J. Lyle, P. Miller, Periocular region appearance cues for biometric identification, in: IEEE International Conference on Computer Vision and Pattern Recognition Workshops (CVPRW), 2010, pp. 162-169.

[41] F. J. Xu, M. Savvides, Subspace-based discrete transform encoded local binary patterns representations for robust periocular matching on NIST's face recog- nition grand challenge, IEEE Trans. Image Process. 23 (2014) 3490-3505.

[42] 197G. Mahalingam, K. Ricanek, Is the eye region more reliable than the face? A preliminary study of face-based recognition on a transgender dataset, in: IEEE Sixth International Conference on Biometrics: Theory, Applications and Systems (BTAS), 2013, pp. 1-7.

[43] 199A. Sharma, S. Verma, M. Vatsa, R. Singh, On cross spectral periocular recog- nition, in: IEEE International Conference on Image Processing (ICIP), 2014, pp. 5007-5011

\section{AUTHORS PROFILE}

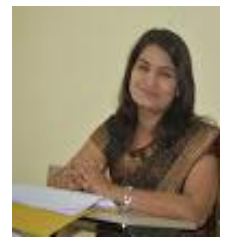

First Author : Ms. Swati D. Shirke holds an M.E.degree in Computer Science and Engineering from Pune University and is a research fellow in the Department of Computer Science and Engineering, Bharath Institute of Higher Education and Research, Bharath University. Her main area of interest includes pattern recognition, image processing, machine learning, She has published several papers in well known peer-reviewed journals.

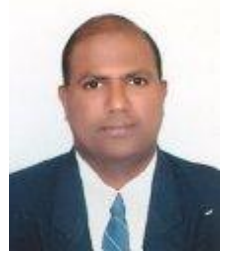

Second Author : Dr. C. Rajabhushnam holds a Ph.D. in Neural Networks, from Louisiana State University, USA And he is a professor in the Department of In the Department of Computer Science and Engineering, Bharath Institute of Higher Education and Research, Bharath University. His main area of interest includes pattern recognition, image processing, and machine learning he has published several papers in well known peer-reviewed journals. 